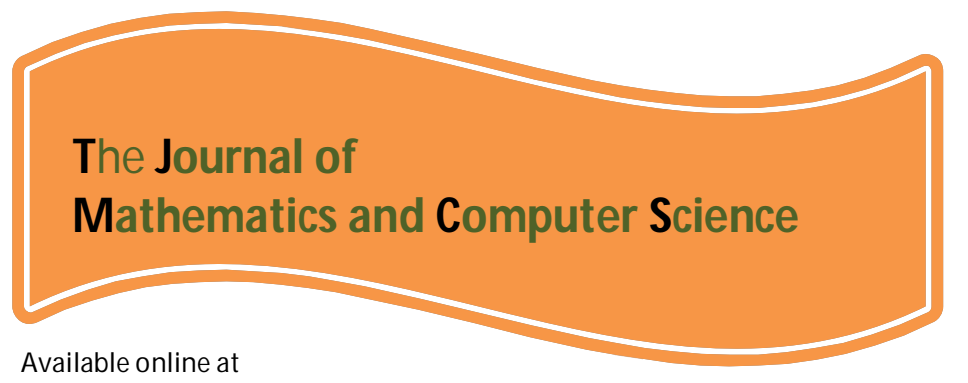

\title{
http://www.TJMCS.com
}

The Journal of Mathematics and Computer Science Vol. 4 No.3 (2012) 301 - 309

\section{On the anisotropic Wiener-Hopf operator, connected with Helmholtze-Sohrodinger equation}

\author{
S.A. Hosseini Matikolai' ${ }^{\text {, H. Jafari², Roshanak Lotfikar }}{ }^{3}$ \\ 1- Department of Mathematics, Yerevan State University, Yerevan, Armenia \\ seyedm895@yahoo.com \\ 2- Department of Mathematics, University of Mazandaran, Babolsar, Iran \\ jafari@umz.ac.ir \\ 3- Islamic Azad University Ilam branch \\ rlotfikar@yahoo.com
}

Received: February 2012, Revised: May 2012

Online Publication: July 2012

\begin{abstract}
In this article, solvability of one the anisotropic Helmholtz-Shrodinger equation with the boundary conditions of the first and second type is investigated in the upper and lower half -space, $\left(\mathrm{x}_{5}>0\right.$, $\mathrm{x}_{5}<0$ ), in 5 dimensions. Solvability of these boundary problems reduces to solvability of RiemanHilbert boundary problem, in general necessary and sufficient conditions for the correctness of the problem in the Sobolev space are presented as well as explicit formulas for a factorization of the Fourier symbol matrix of the one-medium problem. The solvability analysis is based on the factorization problem of some matrix-function. ${ }^{1}$
\end{abstract}

Keywords: Helmholtz-Shrodinger equation, Factorization of matrix-function, Boundary value problem, Wiener- Hopf equation.

\section{Introduction}

Was a certain class of diffraction problems leading investigated to simultaneous $2 \times 2$ systems of Wiener-Hopf equations. First the classical Wiener- Hopf technique was represented by Noble [1]. 


\section{S.A. Hosseini Matikolai, H. Jafari, Roshanak Lotfikar/ TJMCSVol. 4 №. 3 (2012) 301 - 309}

This type of problems studied by A. J. Summerfeld for the wave diffraction on the interface of two media $[2,3]$.

Various physical problems in diffraction theory lead us to study modification of the Sommerfeld half-plane governed by two proper elliptic partial differential equation is complementary $\mathrm{R}^{3}$ halfspace $\Omega^{ \pm}$and allow different boundary or transmission conditions on two half-planes, which together from the common boundary of $\Omega^{ \pm}$[3].

These problems were investigated in the isotropic case $[3,4]$, and studied the problem of finding a function " $u$ " in a suitable space with satisfies [3].

We investigated solvability of the boundary value problem coordinated with the anisotropic Helmholtz- Shrodinger equation:

$$
\left\{\begin{array}{l}
\Delta u+\left(k_{+}^{2}+2 \beta_{+}^{2} \sec h^{2}\left(\beta_{+} y\right)\right) u=0, \text { in } \Omega^{+}, \\
\Delta u+\left(k_{-}^{2}+2 \beta_{-}^{2} \sec h^{2}\left(\beta_{-} y\right)\right) u=0, \text { in } \Omega^{-},
\end{array}\right.
$$

In the Sobolev spaces [5]. Also it was investigated the case of anisotropic Helmholtz-Shrodinger equation (1), where $k_{+}=k_{-}=k$, and the solution of the boundary value problem gained and then prove solvability of this [6]. Also it was surveyed the Fredholmz property of Wiener-Hopf operator for anisotropic boundary value problem for the Helmholtz-Shrodinger equation with the boundary conditions of the first and second type on the line $y=0$ [7].

In this paper we investigate solvability of the boundary value problem coordinated with the anisotropic Helmholtz-Shrodinger equation, in the Sobolev spaces in 5 dimensions. Further we prove that solvability of the boundary value problem is equivalent to solvability of the some Riemann-Hilbert problem in 5 dimensions.

Convention: As a rule, upper or lower indices \pm are related to the half-spaces $\Omega^{ \pm}$except for some standard notation $\mathrm{R}_{ \pm}$and $H^{ \pm \frac{1}{2}}$

\section{Investigate solvability of anisotropic boundary value problem}

Consider the following anistropic Helmholtz-Shrodinger equation $\begin{cases}\Delta u+\left(k_{+}^{2}+2 \beta_{+}^{2} \sec h^{2}\left(\beta_{+} x_{5}\right)\right) u=0, \text { in } \Omega^{+}, \\ \Delta u+\left(k_{-}^{2}+2 \beta_{-}^{2} \sec h^{2}\left(\beta_{-} x_{5}\right)\right) u=0, \text { in } \Omega^{-},\end{cases}$

Let $\Omega^{ \pm}=\left\{\left(x_{1}, x_{2}, \ldots, x_{5}\right) \in R^{5}: x_{5} \lessgtr 0\right\}$ and $\Sigma^{ \pm}=\left\{\left(x_{1}, x_{2}, x_{3}, x_{4}, 0\right) \in R^{4}: x_{4} \gtrless 0\right\}$, where $J_{\mathrm{m}}\left(\mathrm{k}_{ \pm}\right)>0$ and let $H^{\frac{1}{2}}\left(\Omega^{ \pm}\right)$and $H^{-\frac{1}{2}}\left(\Omega^{ \pm}\right)$are the corresponding Sobolev spaces (see [3]). Now we suppose the boundary conditions: 


$$
\left\{\begin{array}{c}
\left\{\begin{array}{l}
a_{0} u\left(x_{1}, x_{2}, x_{3}, x_{4},+0\right)+b_{0} u\left(x_{1}, x_{2}, x_{3}, x_{4},-0\right)=h_{0}\left(x_{1}, x_{2}, x_{3}, x_{4}\right) \\
a_{1} \frac{\partial u\left(x_{1}, x_{2}, x_{3}, x_{4},+0\right)}{\partial x_{5}}+b_{1} \frac{\partial u\left(x_{1}, x_{2}, x_{3}, x_{4},-0\right)}{\partial x_{5}}=h_{1}\left(x_{1}, x_{2}, x_{3}, x_{4}\right) \quad \text { in } \quad \sum^{+}
\end{array}\right. \\
\left\{\begin{array}{l}
c_{0} u\left(x_{1}, x_{2}, x_{3}, x_{4},+0\right)+d_{0} u\left(x_{1}, x_{2}, x_{3}, x_{4},-0\right)=P_{0}\left(x_{1}, x_{2}, x_{3}, x_{4}\right) \\
c_{1} \frac{\partial u\left(x_{1}, x_{2}, x_{3}, x_{4},+0\right)}{\partial x_{5}}+d_{1} \frac{\partial u\left(x_{1}, x_{2}, x_{3}, x_{4},-0\right)}{\partial x_{5}}=P_{1}\left(x_{1}, x_{2}, x_{3}, x_{4}\right) \quad \text { in } \quad \sum^{+}
\end{array}\right.
\end{array}\right.
$$

Where $h_{0} \in H^{\frac{1}{2}}\left(\sum^{+}\right), h_{1} \in H^{-\frac{1}{2}}\left(\sum^{+}\right), p_{0} \in H^{\frac{1}{2}}\left(\sum^{-}\right), p_{1} \in H^{-\frac{1}{2}}\left(\sum^{-}\right) \quad$ and $\mathrm{a}_{0}, \mathrm{~b}_{0}, \mathrm{C}_{0}, \mathrm{~d}_{0}, \mathrm{a}_{1}, \mathrm{~b}_{1}, \mathrm{c}_{1}, \mathrm{~d}_{1}$, are complex constants. For finding the solution of the boundary value problem (2) in the $\mathrm{L}^{2}\left(\mathrm{R}^{5}\right)$, apply Fourier integral transform to the solution $u \in L^{2}\left(R^{5}\right)$ over the variables $x_{1}, x_{2}, x_{3}, x_{4}$, one derives the following system of ordinary differential equations:

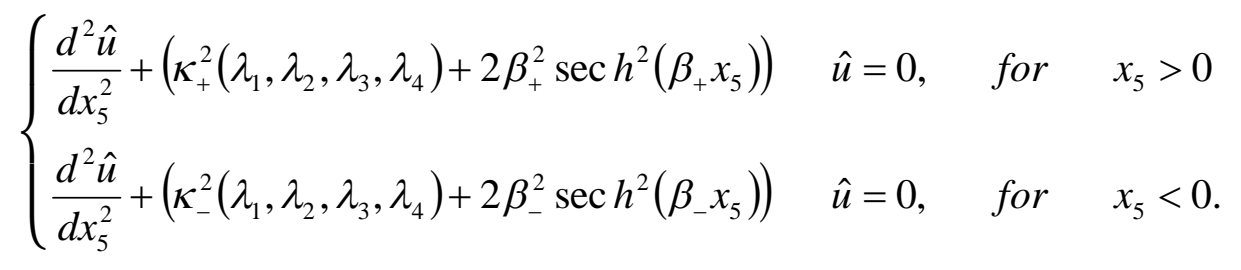

Then $\hat{u} \in L^{2}\left(R^{5}\right)$, we denote $\gamma \pm\left(\lambda_{1}, \lambda_{2}, \lambda_{3}, \lambda_{4}\right)=\sqrt{\sum_{j=1}^{4} \lambda_{j}^{2}-k_{ \pm}^{2}}=i \kappa_{ \pm}\left(\lambda_{1}, \lambda_{2}, \lambda_{3}, \lambda_{4}\right)$.

If follows that the general solutions of the system of ordinary differential equations (4) in the $L^{2}\left(R^{5}\right)$-space has the following form:

$$
\hat{u}\left(\lambda_{1}, \lambda_{2}, \lambda_{3}, \lambda_{4}, x_{5}\right)=\left\{\begin{array}{l}
a\left(\lambda_{1}, \lambda_{2}, \lambda_{3}, \lambda_{4}\right) \frac{i \kappa_{+}\left(\lambda_{1}, \lambda_{2}, \lambda_{3}, \lambda_{4}\right)-\beta_{+} \tanh \left(\beta_{+} x_{5}\right)}{i \kappa_{+}\left(\lambda_{1}, \lambda_{2}, \lambda_{3}, \lambda_{4}\right)}\left(e^{i \kappa_{+}\left(\lambda_{1}, \lambda_{2}, \lambda_{3}, \lambda_{4}\right) x_{5}}\right), x_{5}>0 \\
b\left(\lambda_{1}, \lambda_{2}, \lambda_{3}, \lambda_{4}\right) \frac{i \kappa_{-}\left(\lambda_{1}, \lambda_{2}, \lambda_{3}, \lambda_{4}\right)+\beta_{-} \tanh \left(\beta_{-} x_{5}\right)}{i \kappa_{-}\left(\lambda_{1}, \lambda_{2}, \lambda_{3}, \lambda_{4}\right)}\left(e^{\left.-i \kappa_{-}\left(\lambda_{1}, \lambda_{2}, \lambda_{3}, \lambda_{4}\right) x_{5}\right), x_{5}<0}\right.
\end{array}\right.
$$

Let $\chi_{ \pm}\left(x_{5}\right)=1 / 2\left(\operatorname{sgn} x_{5}\right)$, and

$$
\left\{\begin{array}{l}
\hat{u}_{+}\left(\lambda_{1}, \lambda_{2}, \lambda_{3}, \lambda_{4}, x_{5}\right)=\frac{\chi_{+}\left(x_{5}\right)}{(2 \pi)^{2}} \int_{-\infty}^{+\infty} \int_{-\infty}^{+\infty} \ldots \int_{-\infty}^{+\infty} u\left(x_{1}, x_{2}, \ldots, x_{5}\right) e^{i \sum_{K=1}^{4} \lambda_{k} x_{k}} d x_{1} d x_{2} \ldots d x_{5} \\
\hat{u}_{-}\left(\lambda_{1}, \lambda_{2}, \lambda_{3}, \lambda_{4}, x_{5}\right)=\frac{\chi_{-}\left(x_{5}\right)}{(2 \pi)^{2}} \int_{-\infty}^{+\infty} \int_{-\infty}^{+\infty} \ldots \int_{-\infty}^{+\infty} u\left(x_{1}, x_{2}, \ldots, x_{5}\right) e^{i \sum_{K=1}^{4} \lambda_{k} x_{k}} d x_{1} d x_{2} \ldots d x_{5}
\end{array}\right.
$$

Then from equation (5) it follows that

$$
\hat{u}\left(\lambda_{1}, \lambda_{2}, \lambda_{3}, \lambda_{4}, x_{5}\right)=\hat{u}_{+}\left(\lambda_{1}, \lambda_{2}, \lambda_{3}, \lambda_{4}, x_{5}\right)+\hat{u}_{-}\left(\lambda_{1}, \lambda_{2}, \lambda_{3}, \lambda_{4}, x_{5}\right)
$$


S.A. Hosseini Matikolai, H. Jafari, Roshanak Lotfikar/ TJMCSVol. 4 №. 3 (2012) 301 - 309

We introduce the following notations:

$$
\left\{\begin{array}{l}
u_{-}\left(\lambda_{1}, \lambda_{2}, \lambda_{3}, \lambda_{4}\right)=\frac{1}{(2 \pi)^{2}} \int_{-\infty}^{0} \int_{-\infty}^{0} \cdots \int_{-\infty}^{0}\left(a_{0} u\left(x_{1}, x_{2}, x_{3}, x_{4},+0\right)\right. \\
\left.+b_{0} u\left(x_{1}, x_{2}, x_{3}, x_{4},-0\right)-h_{0}\left(x_{1}, x_{2}, x_{3}, x_{4}\right)\right) e^{i \sum_{k=1}^{4} \lambda_{k} x_{k}} d x_{1} d x_{2} d x_{3} d x_{4} \\
w_{-}\left(\lambda_{1}, \lambda_{2}, \lambda_{3}, \lambda_{4}\right)=\frac{1}{(2 \pi)^{2}} \int_{-\infty}^{0} \int_{-\infty}^{0} \cdots \int_{-\infty}^{0}\left(a_{1} \frac{\partial u\left(x_{1}, x_{2}, x_{3}, x_{4},+0\right)}{\partial x_{5}}+b_{1} \frac{\partial u\left(x_{1}, x_{2}, x_{3}, x_{4},-0\right)}{\partial x_{5}}\right. \\
\left.-h_{1}\left(x_{1}, x_{2}, x_{3}, x_{4}\right)\right) e^{i \sum_{k=1}^{4} \lambda_{k} x_{k}} d x_{1} d x_{2} d x_{3} d x_{4} .
\end{array}\right.
$$

Similarly

$$
\left\{\begin{array}{l}
u_{+}\left(\lambda_{1}, \lambda_{2}, \lambda_{3}, \lambda_{4}\right)=\frac{1}{(2 \pi)^{2}} \int_{0}^{+\infty} \int_{0}^{+\infty} \cdots \int_{0}^{+\infty}\left(c_{0} u\left(x_{1}, x_{2}, x_{3}, x_{4},+0\right)\right. \\
\left.+d_{0} u\left(x_{1}, x_{2}, x_{3}, x_{4},-0\right)-p_{0}\left(x_{1}, x_{2}, x_{3}, x_{4}\right)\right) e^{i \sum_{k=1}^{4} \lambda_{k} x_{k}} d x_{1} d x_{2} d x_{3} d x_{4}, \\
w_{+}\left(\lambda_{1}, \lambda_{2}, \lambda_{3}, \lambda_{4}\right)=\frac{1}{(2 \pi)^{2}} \int_{0}^{+\infty} \int_{0}^{+\infty} \cdots \int_{0}^{+\infty}\left(c_{1} \frac{\partial u\left(x_{1}, x_{2}, x_{3}, x_{4},+0\right)}{\partial x_{5}}+d_{1} \frac{\partial u\left(x_{1}, x_{2}, x_{3}, x_{4},-0\right)}{\partial x_{5}}\right. \\
\left.-p_{1}\left(x_{1}, x_{2}, x_{3}, x_{4}\right)\right) e^{i \sum_{k=1}^{4} \lambda_{k} x_{k}} d x_{1} d x_{2} d x_{3} d x_{4} .
\end{array}\right.
$$

So

$$
\frac{\partial \hat{u}\left(x_{1}, x_{2}, x_{3}, x_{4}, x_{5}\right)}{\partial x_{5}}=\left\{\begin{array}{l}
a\left(\lambda_{1}, \lambda_{2}, \lambda_{3}, \lambda_{4}\right)\left[i \kappa_{+}\left(\lambda_{1}, \lambda_{2}, \lambda_{3}, \lambda_{4}\right)-\beta_{+} \tanh \left(\beta_{+} x_{5}\right)-\frac{\beta_{+}{ }^{2}}{i \kappa_{+}\left(\lambda_{1}, \lambda_{2}, \lambda_{3}, \lambda_{4}\right) \cosh ^{2}\left(\beta_{+} x_{5}\right)}\right] \\
\left(e^{i \kappa_{+}\left(\lambda_{1}, \lambda_{2}, \lambda_{3}, \lambda_{4}\right) x_{5}}\right), x_{5}>0 \\
-b\left(\lambda_{1}, \lambda_{2}, \lambda_{3}, \lambda_{4}\right)\left[i \kappa_{-}\left(\lambda_{1}, \lambda_{2}, \lambda_{3}, \lambda_{4}\right)+\beta_{-} \tanh \left(\beta_{-} x_{5}\right)+\frac{\beta_{-}^{2}}{i \kappa_{-}\left(\lambda_{1}, \lambda_{2}, \lambda_{3}, \lambda_{4}\right) \cosh ^{2}\left(\beta_{-} x_{5}\right)}\right] \\
\left(e^{-i \kappa_{-}\left(\lambda_{1}, \lambda_{2}, \lambda_{3}, \lambda_{4}\right) x_{5}}\right), x_{5}<0 .
\end{array}\right.
$$

Using boundary conditions (3) and taking into account equations (5), (10) one derives:

$$
\left\{\begin{array}{l}
a_{0} a\left(\lambda_{1}, \lambda_{2}, \lambda_{3}, \lambda_{4}\right)+b_{0} b\left(\lambda_{1}, \lambda_{2}, \lambda_{3}, \lambda_{4}\right)=u_{-}\left(\lambda_{1}, \lambda_{2}, \lambda_{3}, \lambda_{4}\right)+\hat{h}_{0}\left(\lambda_{1}, \lambda_{2}, \lambda_{3}, \lambda_{4}\right) \\
\frac{-a_{1}\left[\kappa_{+}^{2}\left(\lambda_{1}, \lambda_{2}, \lambda_{3}, \lambda_{4}\right)+\beta_{+}^{2}\right] a\left(\lambda_{1}, \lambda_{2}, \lambda_{3}, \lambda_{4}\right)}{i \kappa_{+}\left(\lambda_{1}, \lambda_{2}, \lambda_{3}, \lambda_{4}\right)}+\frac{b_{1}\left[\kappa_{-}^{2}\left(\lambda_{1}, \lambda_{2}, \lambda_{3}, \lambda_{4}\right)+\beta_{-}^{2}\right] b\left(\lambda_{1}, \lambda_{2}, \lambda_{3}, \lambda_{4}\right)}{i \kappa_{-}\left(\lambda_{1}, \lambda_{2}, \lambda_{3}, \lambda_{4}\right)} \\
=w_{-}\left(\lambda_{1}, \lambda_{2}, \lambda_{3}, \lambda_{4}\right)+\hat{h}_{0}\left(\lambda_{1}, \lambda_{2}, \lambda_{3}, \lambda_{4}\right)
\end{array}\right.
$$

Where 


$$
\left\{\begin{array}{l}
\hat{h}_{0}\left(\lambda_{1}, \lambda_{2}, \lambda_{3}, \lambda_{4}\right)=\frac{1}{(2 \pi)^{2}} \int_{-\infty}^{0} \int_{-\infty}^{0} \ldots \int_{-\infty}^{0} h_{0}\left(x_{1}, x_{2}, x_{3}, x_{4}\right) e^{i \sum_{k=1}^{4} \lambda_{k} x_{k}} d x_{1} d x_{2} d x_{3} d x_{4} \\
\hat{h}_{1}\left(\lambda_{1}, \lambda_{2}, \lambda_{3}, \lambda_{4}\right)=\frac{1}{(2 \pi)^{2}} \int_{-\infty}^{0} \int_{-\infty}^{0} \ldots \int_{-\infty}^{0} h_{1}\left(x_{1}, x_{2}, x_{3}, x_{4}\right) e^{i \sum_{k=1}^{4} \lambda_{k} x_{k}} d x_{1} d x_{2} d x_{3} d x_{4}
\end{array}\right.
$$

Assume that the determinant $\Delta\left(\lambda_{1}, \lambda_{2}, \lambda_{3}, \lambda_{4}\right)$, of system (11), is not zero, i.e.

$$
\begin{aligned}
& \Delta\left(\lambda_{1}, \lambda_{2}, \lambda_{3}, \lambda_{4}\right)=a_{0} b_{1} \frac{\kappa_{-}^{2}\left(\lambda_{1}, \lambda_{2}, \lambda_{3}, \lambda_{4}\right)+\beta_{-}^{2}}{i \kappa_{-}\left(\lambda_{1}, \lambda_{2}, \lambda_{3}, \lambda_{4}\right)}+a_{1} b_{0} \frac{\kappa_{+}^{2}\left(\lambda_{1}, \lambda_{2}, \lambda_{3}, \lambda_{4}\right)+\beta_{+}^{2}}{i \kappa_{+}\left(\lambda_{1}, \lambda_{2}, \lambda_{3}, \lambda_{4}\right)} \\
& =a_{0} b_{1} \frac{\gamma_{-}^{2}\left(\lambda_{1}, \lambda_{2}, \lambda_{3}, \lambda_{4}\right)-\beta_{-}^{2}}{\gamma_{-}\left(\lambda_{1}, \lambda_{2}, \lambda_{3}, \lambda_{4}\right)}+a_{1} b_{0} \frac{\gamma_{+}^{2}\left(\lambda_{1}, \lambda_{2}, \lambda_{3}, \lambda_{4}\right)-\beta_{+}^{2}}{\gamma_{+}\left(\lambda_{1}, \lambda_{2}, \lambda_{3}, \lambda_{4}\right)} \neq 0
\end{aligned}
$$

In view of equation (11):

$$
\left\{\begin{array}{l}
a\left(\lambda_{1}, \lambda_{2}, \lambda_{3}, \lambda_{4}\right)=\frac{1}{\Delta\left(\lambda_{1}, \lambda_{2}, \lambda_{3}, \lambda_{4}\right)}\left\{b_{1} \frac{\kappa_{-}^{2}\left(\lambda_{1}, \lambda_{2}, \lambda_{3}, \lambda_{4}\right)+\beta_{-}^{2}}{i \kappa_{-}\left(\lambda_{1}, \lambda_{2}, \lambda_{3}, \lambda_{4}\right)}\left(u_{-}\left(\lambda_{1}, \lambda_{2}, \lambda_{3}, \lambda_{4}\right)+\hat{h}_{0}\left(\lambda_{1}, \lambda_{2}, \lambda_{3}, \lambda_{4}\right)\right)\right\} \\
-\frac{1}{\Delta\left(\lambda_{1}, \lambda_{2}, \lambda_{3}, \lambda_{4}\right)}\left\{b_{0}\left(w_{-}\left(\lambda_{1}, \lambda_{2}, \lambda_{3}, \lambda_{4}\right)+\hat{h}_{1}\left(\lambda_{1}, \lambda_{2}, \lambda_{3}, \lambda_{4}\right)\right)\right\} \\
b\left(\lambda_{1}, \lambda_{2}, \lambda_{3}, \lambda_{4}\right)=\frac{1}{\Delta\left(\lambda_{1}, \lambda_{2}, \lambda_{3}, \lambda_{4}\right)}\left\{a_{1} \frac{\kappa_{-}^{2}\left(\lambda_{1}, \lambda_{2}, \lambda_{3}, \lambda_{4}\right)+\beta_{-}^{2}}{i \kappa_{-}\left(\lambda_{1}, \lambda_{2}, \lambda_{3}, \lambda_{4}\right)}\left(u_{-}\left(\lambda_{1}, \lambda_{2}, \lambda_{3}, \lambda_{4}\right)+\hat{h}_{0}\left(\lambda_{1}, \lambda_{2}, \lambda_{3}, \lambda_{4}\right)\right)\right\} \\
+\frac{1}{\Delta\left(\lambda_{1}, \lambda_{2}, \lambda_{3}, \lambda_{4}\right)}\left\{a_{0}\left(w_{-}\left(\lambda_{1}, \lambda_{2}, \lambda_{3}, \lambda_{4}\right)+\hat{h}_{1}\left(\lambda_{1}, \lambda_{2}, \lambda_{3}, \lambda_{4}\right)\right)\right\}
\end{array}\right.
$$

Then taking into account that

$$
\left\{\begin{array}{l}
u_{+}\left(\lambda_{1}, \lambda_{2}, \lambda_{3}, \lambda_{4}\right)=c_{0} a\left(\lambda_{1}, \lambda_{2}, \lambda_{3}, \lambda_{4}\right)+d_{0} b\left(\lambda_{1}, \lambda_{2}, \lambda_{3}, \lambda_{4}\right)-\hat{p}_{0}\left(\lambda_{1}, \lambda_{2}, \lambda_{3}, \lambda_{4}\right) \\
w_{+}\left(\lambda_{1}, \lambda_{2}, \lambda_{3}, \lambda_{4}\right)=\frac{-c_{1}\left[\kappa_{+}^{2}\left(\lambda_{1}, \lambda_{2}, \lambda_{3}, \lambda_{4}\right)+\beta_{+}^{2}\right] a\left(\lambda_{1}, \lambda_{2}, \lambda_{3}, \lambda_{4}\right)}{i \kappa_{+}\left(\lambda_{1}, \lambda_{2}, \lambda_{3}, \lambda_{4}\right)} \\
+\frac{d_{1}\left[\kappa_{-}^{2}\left(\lambda_{1}, \lambda_{2}, \lambda_{3}, \lambda_{4}\right)+\beta_{-}^{2}\right] b\left(\lambda_{1}, \lambda_{2}, \lambda_{3}, \lambda_{4}\right)}{i \kappa_{-}\left(\lambda_{1}, \lambda_{2}, \lambda_{3}, \lambda_{4}\right)}-\hat{p}_{1}\left(\lambda_{1}, \lambda_{2}, \lambda_{3}, \lambda_{4}\right)
\end{array}\right.
$$

Where

$$
\left\{\begin{array}{l}
\hat{p}_{0}\left(\lambda_{1}, \lambda_{2}, \lambda_{3}, \lambda_{4}\right)=\frac{1}{(2 \pi)^{2}} \int_{0}^{+\infty} \int_{0}^{+\infty} \ldots \int_{0}^{+\infty} \hat{p}_{0}\left(x_{1}, x_{2}, x_{3}, x_{4}\right) e^{i \sum_{k=1}^{4} \lambda_{k} x_{k}} d x_{1} d x_{2} d x_{3} d x_{4} \\
\hat{p}_{1}\left(\lambda_{1}, \lambda_{2}, \lambda_{3}, \lambda_{4}\right)=\frac{1}{(2 \pi)^{2}} \int_{-\infty}^{0} \int_{0}^{+\infty} \cdots \int_{-\infty}^{0} \hat{p}_{1}\left(x_{1}, x_{2}, x_{3}, x_{4}\right) e^{i \sum_{k=1}^{4} \lambda_{k} x_{k}} d x_{1} d x_{2} d x_{3} d x_{4}
\end{array}\right.
$$

Which derives the following boundary problem of Riemann-Hilbert with respect to 
$\vec{u}_{+}\left(\lambda_{1}, \lambda_{2}, \lambda_{3}, \lambda_{4}\right)=\left(\begin{array}{l}u_{+}\left(\lambda_{1}, \lambda_{2}, \lambda_{3}, \lambda_{4}\right) \\ w_{-}\left(\lambda_{1}, \lambda_{2}, \lambda_{3}, \lambda_{4}\right)\end{array}\right), \quad \vec{u}_{-}\left(\lambda_{1}, \lambda_{2}, \lambda_{3}, \lambda_{4}\right)=\left(\begin{array}{c}u_{+}\left(\lambda_{1}, \lambda_{2}, \lambda_{3}, \lambda_{4}\right) \\ w_{+}\left(\lambda_{1}, \lambda_{2}, \lambda_{3}, \lambda_{4}\right)\end{array}\right)$,

$\vec{u}_{+}\left(\lambda_{1}, \lambda_{2}, \lambda_{3}, \lambda_{4}\right)$ and $\vec{u}_{-}\left(\lambda_{1}, \lambda_{2}, \lambda_{3}, \lambda_{4}\right)$ are analytical functions respectively in the vector notations this problem takes the following form

$\vec{u}_{+}\left(\lambda_{1}, \lambda_{2}, \lambda_{3}, \lambda_{4}\right)=L\left(\lambda_{1}, \lambda_{2}, \lambda_{3}, \lambda_{4}\right) \vec{u}_{-}\left(\lambda_{1}, \lambda_{2}, \lambda_{3}, \lambda_{4}\right)+\vec{m}\left(\lambda_{1}, \lambda_{2}, \lambda_{3}, \lambda_{4}\right)$

Where the matrix function $L\left(\lambda_{1}, \lambda_{2}, \lambda_{3}, \lambda_{4}\right)$ is:

$L\left(\lambda_{1}, \lambda_{2}, \lambda_{3}, \lambda_{4}\right)=\frac{1}{\Delta\left(\lambda_{1}, \lambda_{2}, \lambda_{3}, \lambda_{4}\right)}\left(\begin{array}{ll}A_{11}\left(\lambda_{1}, \lambda_{2}, \lambda_{3}, \lambda_{4}\right), & A_{12}\left(\lambda_{1}, \lambda_{2}, \lambda_{3}, \lambda_{4}\right) \\ A_{21}\left(\lambda_{1}, \lambda_{2}, \lambda_{3}, \lambda_{4}\right), & A_{22}\left(\lambda_{1}, \lambda_{2}, \lambda_{3}, \lambda_{4}\right)\end{array}\right)$

With

$$
\left\{\begin{array}{l}
A_{11}\left(\lambda_{1}, \lambda_{2}, \lambda_{3}, \lambda_{4}\right)=a_{1} d_{0} \frac{\kappa_{+}^{2}\left(\lambda_{1}, \lambda_{2}, \lambda_{3}, \lambda_{4}\right)+\beta_{+}^{2}}{i \kappa_{+}\left(\lambda_{1}, \lambda_{2}, \lambda_{3}, \lambda_{4}\right)}+b_{1} c_{0} \frac{\kappa_{-}^{2}\left(\lambda_{1}, \lambda_{2}, \lambda_{3}, \lambda_{4}\right)+\beta_{-}^{2}}{i \kappa_{-}\left(\lambda_{1}, \lambda_{2}, \lambda_{3}, \lambda_{4}\right)} \\
=a_{1} d_{0} \frac{\gamma_{+}^{2}\left(\lambda_{1}, \lambda_{2}, \lambda_{3}, \lambda_{4}\right)-\beta_{+}^{2}}{\gamma_{+}\left(\lambda_{1}, \lambda_{2}, \lambda_{3}, \lambda_{4}\right)}+b_{1} c_{0} \frac{\gamma_{-}^{2}\left(\lambda_{1}, \lambda_{2}, \lambda_{3}, \lambda_{4}\right)-\beta_{-}^{2}}{\gamma_{+}\left(\lambda_{1}, \lambda_{2}, \lambda_{3}, \lambda_{4}\right)} \\
A_{12}\left(\lambda_{1}, \lambda_{2}, \lambda_{3}, \lambda_{4}\right)=a_{0} d_{0}-b_{0} c_{o} \\
A_{21}\left(\lambda_{1}, \lambda_{2}, \lambda_{3}, \lambda_{4}\right)=\left(a_{1} d_{1}-b_{1} c_{1}\right) \frac{\kappa_{+}^{2}\left(\lambda_{1}, \lambda_{2}, \lambda_{3}, \lambda_{4}\right)+\beta_{+}^{2}}{i \kappa_{+}\left(\lambda_{1}, \lambda_{2}, \lambda_{3}, \lambda_{4}\right)} \times \frac{\kappa_{-}^{2}\left(\lambda_{1}, \lambda_{2}, \lambda_{3}, \lambda_{4}\right)+\beta_{-}^{2}}{i \kappa_{-}\left(\lambda_{1}, \lambda_{2}, \lambda_{3}, \lambda_{4}\right)} \\
=\left(a_{1} d_{1}-b_{1} c_{1}\right) \frac{\gamma_{+}^{2}\left(\lambda_{1}, \lambda_{2}, \lambda_{3}, \lambda_{4}\right)-\beta_{+}^{2}}{\gamma_{+}\left(\lambda_{1}, \lambda_{2}, \lambda_{3}, \lambda_{4}\right)} \times \frac{\gamma_{-}^{2}\left(\lambda_{1}, \lambda_{2}, \lambda_{3}, \lambda_{4}\right)-\beta_{-}^{2}}{\gamma_{-}\left(\lambda_{1}, \lambda_{2}, \lambda_{3}, \lambda_{4}\right)} \\
A_{22}\left(\lambda_{1}, \lambda_{2}, \lambda_{3}, \lambda_{4}\right)=b_{0} c_{1} \frac{\kappa_{+}^{2}\left(\lambda_{1}, \lambda_{2}, \lambda_{3}, \lambda_{4}\right)+\beta_{+}^{2}}{i \kappa_{+}\left(\lambda_{1}, \lambda_{2}, \lambda_{3}, \lambda_{4}\right)}+a_{0} d_{1} \frac{\kappa_{-}^{2}\left(\lambda_{1}, \lambda_{2}, \lambda_{3}, \lambda_{4}\right)+\beta_{-}^{2}}{i \kappa_{-}\left(\lambda_{1}, \lambda_{2}, \lambda_{3}, \lambda_{4}\right)} \\
=b_{0} c_{1} \frac{\gamma_{+}^{2}\left(\lambda_{1}, \lambda_{2}, \lambda_{3}, \lambda_{4}\right)-\beta_{+}^{2}}{\gamma_{+}\left(\lambda_{1}, \lambda_{2}, \lambda_{3}, \lambda_{4}\right)}+a_{0} d_{1} \frac{\gamma_{-}^{2}\left(\lambda_{1}, \lambda_{2}, \lambda_{3}, \lambda_{4}\right)-\beta_{-}^{2}}{\gamma_{-}\left(\lambda_{1}, \lambda_{2}, \lambda_{3}, \lambda_{4}\right)}
\end{array}\right.
$$

The coordinates of the vector-function $\vec{m}\left(\lambda_{1}, \lambda_{2}, \lambda_{3}, \lambda_{4}\right)$

$\vec{m}\left(\lambda_{1}, \lambda_{2}, \lambda_{3}, \lambda_{4}\right)=\left(\begin{array}{l}m_{1}\left(\lambda_{1}, \lambda_{2}, \lambda_{3}, \lambda_{4}\right) \\ m_{2}\left(\lambda_{1}, \lambda_{2}, \lambda_{3}, \lambda_{4}\right)\end{array}\right)$

Have the following form 


$$
\left\{\begin{array}{l}
m_{1}\left(\lambda_{1}, \lambda_{2}, \lambda_{3}, \lambda_{4}\right)=\frac{\hat{h}_{0}\left(\lambda_{1}, \lambda_{2}, \lambda_{3}, \lambda_{4}\right)}{\Delta\left(\lambda_{1}, \lambda_{2}, \lambda_{3}, \lambda_{4}\right)}\left\{a_{1} d_{0} \frac{\kappa_{+}^{2}\left(\lambda_{1}, \lambda_{2}, \lambda_{3}, \lambda_{4}\right)+\beta_{+}^{2}}{i \kappa_{+}\left(\lambda_{1}, \lambda_{2}, \lambda_{3}, \lambda_{4}\right)}+b_{1} c_{0} \frac{\kappa_{-}^{2}\left(\lambda_{1}, \lambda_{2}, \lambda_{3}, \lambda_{4}\right)+\beta_{-}^{2}}{i \kappa_{-}\left(\lambda_{1}, \lambda_{2}, \lambda_{3}, \lambda_{4}\right)}\right\} \\
+\frac{a_{0} d_{0}-b_{0} c_{0}}{\Delta\left(\lambda_{1}, \lambda_{2}, \lambda_{3}, \lambda_{4}\right)} \hat{h}_{1}\left(\lambda_{1}, \lambda_{2}, \lambda_{3}, \lambda_{4}\right)-\hat{p}_{0}\left(\lambda_{1}, \lambda_{2}, \lambda_{3}, \lambda_{4}\right) \\
m_{2}\left(\lambda_{1}, \lambda_{2}, \lambda_{3}, \lambda_{4}\right)=\frac{\hat{h}_{1}\left(\lambda_{1}, \lambda_{2}, \lambda_{3}, \lambda_{4}\right)}{\Delta\left(\lambda_{1}, \lambda_{2}, \lambda_{3}, \lambda_{4}\right)}\left\{b_{0} c_{1} \frac{\kappa_{+}^{2}\left(\lambda_{1}, \lambda_{2}, \lambda_{3}, \lambda_{4}\right)+\beta_{+}^{2}}{i \kappa_{+}\left(\lambda_{1}, \lambda_{2}, \lambda_{3}, \lambda_{4}\right)}+a_{0} d_{1} \frac{\kappa_{-}^{2}\left(\lambda_{1}, \lambda_{2}, \lambda_{3}, \lambda_{4}\right)+\beta_{-}^{2}}{i \kappa_{-}\left(\lambda_{1}, \lambda_{2}, \lambda_{3}, \lambda_{4}\right)}\right\} \\
+\frac{a_{1} d_{1}-b_{1} c_{1}}{\Delta\left(\lambda_{1}, \lambda_{2}, \lambda_{3}, \lambda_{4}\right)} \hat{h}_{0}\left(\lambda_{1}, \lambda_{2}, \lambda_{3}, \lambda_{4}\right) \frac{\kappa_{+}^{2}\left(\lambda_{1}, \lambda_{2}, \lambda_{3}, \lambda_{4}\right)+\beta_{+}^{2}}{i \kappa_{+}\left(\lambda_{1}, \lambda_{2}, \lambda_{3}, \lambda_{4}\right)} \times \frac{\kappa_{-}^{2}\left(\lambda_{1}, \lambda_{2}, \lambda_{3}, \lambda_{4}\right)+\beta_{-}^{2}}{i \kappa_{-}\left(\lambda_{1}, \lambda_{2}, \lambda_{3}, \lambda_{4}\right)}-\hat{p}_{1}\left(\lambda_{1}, \lambda_{2}, \lambda_{3}, \lambda_{4}\right)
\end{array}\right.
$$

For find the function $u \in L^{2}\left(R^{5}\right)$ for the anisotropic boundary problem $(\mathcal{A P})$ in space $\mathrm{R}^{5}$, with respect to vector-functions $\left\{\overrightarrow{u_{+}}\left(\lambda_{1}, \lambda_{2}, \lambda_{3}, \lambda_{4}\right), \overrightarrow{u_{-}}\left(\lambda_{1}, \lambda_{2}, \lambda_{3}, \lambda_{4}\right)\right\}$, one derives the following Riemann-Hilbert boundary problem (16), satisfies in $\hat{H}^{\frac{1}{2}}\left(\Sigma^{+}\right) \times \hat{H}^{-\frac{1}{2}}\left(\Sigma^{-}\right)$.

Therefore vector-functions $\left\{\overrightarrow{u_{+}}\left(\lambda_{1}, \lambda_{2}, \lambda_{3}, \lambda_{4}\right), \overrightarrow{u_{-}}\left(\lambda_{1}, \lambda_{2}, \lambda_{3}, \lambda_{4}\right)\right\}$, with notice to (16), we take $\hat{u}_{+}\left(\lambda_{1}, \lambda_{2}, \lambda_{3}, \lambda_{4}, 0\right)=a\left(\lambda_{1}, \lambda_{2}, \lambda_{3}, \lambda_{4}\right)$, and $\hat{u}_{-}\left(\lambda_{1}, \lambda_{2}, \lambda_{3}, \lambda_{4}, 0\right)=b\left(\lambda_{1}, \lambda_{2}, \lambda_{3}, \lambda_{4}\right)$, then taking into account that

$$
\left\{\begin{array}{l}
u^{+}\left(x_{1}, x_{2}, x_{3}, x_{4}, x_{5}\right)=F_{\left(\lambda_{1}, \lambda_{2}, \lambda_{3}, \lambda_{4}\right) \rightarrow\left(x_{1}, x_{2}, x_{3}, x_{4}\right)}^{-1} \hat{u}_{+}\left(\lambda_{1}, \lambda_{2}, \lambda_{3}, \lambda_{4}, x_{5}\right) \\
=F_{\left(\lambda_{1}, \lambda_{2}, \lambda_{3}, \lambda_{4}\right) \rightarrow\left(x_{1}, x_{2}, x_{3}, x_{4}\right)}^{-1}\left\{e^{-x_{5} \gamma_{+}\left(\xi_{1}, \zeta_{2}, \xi_{3}, \xi_{4}\right)} \hat{u}_{+}\left(\lambda_{1}, \lambda_{2}, \lambda_{3}, \lambda_{4}, 0\right) \chi_{+}\left(x_{5}\right)\right\} \in H^{1}\left(\Omega^{+}\right) \\
u^{-}\left(x_{1}, x_{2}, x_{3}, x_{4}, x_{5}\right)=F_{\left(\lambda_{1}, \lambda_{2}, \lambda_{3}, \lambda_{4}\right) \rightarrow\left(x_{1}, x_{2}, x_{3}, x_{4}\right)}^{-1} \hat{u}_{-}\left(\lambda_{1}, \lambda_{2}, \lambda_{3}, \lambda_{4}, x_{5}\right) \\
=F_{\left(\lambda_{1}, \lambda_{2}, \lambda_{3}, \lambda_{4}\right) \rightarrow\left(x_{1}, x_{2}, x_{3}, x_{4}\right)}^{-1}\left\{e^{-x_{5} \gamma_{+}\left(\xi_{1}, \zeta_{2}, \xi_{3}, \xi_{4}\right)} \hat{u}_{-}\left(\lambda_{1}, \lambda_{2}, \lambda_{3}, \lambda_{4}, 0\right) \chi_{-}\left(x_{5}\right)\right\} \in H^{1}\left(\Omega^{-}\right)
\end{array}\right.
$$

And function

$$
u\left(x_{1}, x_{2}, x_{3}, x_{4}, x_{5}\right)=u^{+}\left(x_{1}, x_{2}, x_{3}, x_{4}, x_{5}\right)+u^{-}\left(x_{1}, x_{2}, x_{3}, x_{4}, x_{5}\right)
$$

Is the Solution anisotropic boundary problem $(\mathcal{A P})$

In this article we have this result:

Theorem: If the function $u \in L^{2}\left(R^{5}\right)$ is the solution of the boundary problem (2), then the pair of vector -functions $\left\{\overrightarrow{u_{+}}\left(\lambda_{1}, \lambda_{2}, \lambda_{3}, \lambda_{4}\right), \overrightarrow{u_{-}}\left(\lambda_{1}, \lambda_{2}, \lambda_{3}, \lambda_{4}\right)\right\}$ is a solution of the boundary problem of Riemann-Hilbert (16). Vice-versa, if applies inverse Fourier transform which is associated with vector-functions $\left\{\overrightarrow{u_{+}}\left(\lambda_{1}, \lambda_{2}, \lambda_{3}, \lambda_{4}\right), \overrightarrow{u_{-}}\left(\lambda_{1}, \lambda_{2}, \lambda_{3}, \lambda_{4}\right)\right\}$, the function 
S.A. Hosseini Matikolai, H. Jafari, Roshanak Lotfikar/ TJMCSVol. 4 №. 3 (2012) 301 - 309

$$
\begin{gathered}
\left.u\left(x_{1}, x_{2}, x_{3}, x_{4}, x_{5}\right)=F_{\left(\lambda_{1}, \lambda_{2}, \lambda_{3}, \lambda_{4}\right) \rightarrow\left(x_{1}, x_{2}, x_{3}, x_{4}\right)}^{-1} \overrightarrow{u_{+}}\left(\lambda_{1}, \lambda_{2}, \lambda_{3}, \lambda_{4}\right)+\overrightarrow{u_{-}}\left(\lambda_{1}, \lambda_{2}, \lambda_{3}, \lambda_{4}\right)\right\} \\
=u^{+}\left(x_{1}, x_{2}, x_{3}, x_{4}, x_{5}\right)+u^{-}\left(x_{1}, x_{2}, x_{3}, x_{4}, x_{5}\right)=F^{-1}\left(\lambda_{1}, \lambda_{2}, \lambda_{3}, \lambda_{4}\right) \rightarrow\left(x_{1}, x_{2}, x_{3}, x_{4}\right) \\
\left\{e^{-x_{5} \gamma_{+}\left(\xi_{1}, \xi_{2}, \xi_{3}, \xi_{4}\right)} \widehat{u_{+}}\left(\lambda_{1}, \lambda_{2}, \lambda_{3}, \lambda_{4}, 0\right) \chi_{+}\left(x_{5}\right)+e^{-x_{5} \gamma_{+}\left(\xi_{1}, \xi_{2}, \xi_{3}, \xi_{4}\right)} \widehat{u_{-}}\left(\lambda_{1}, \lambda_{2}, \lambda_{3}, \lambda_{4}, 0\right) \chi_{-}\left(x_{5}\right)\right\} .
\end{gathered}
$$

By the case of relations (8), (9), the solutions of the boundary problem (2), will be derived.A The case of $\beta_{-}=\beta_{+}=0$ was studied in the papers [2,3].

\section{References}

[ 1] B. Noble. Methods Based on the Wiener- Hopf Technique for the Solution of Partial Differential Equations, Pergamon, London, P.P. 223-230(1958)

[ 2] A.D. Rawlins. The Explicit Wiener - Hopf Factorization of a Special Matrix. Z. Angew, Math. Mech., 61, p.p. 527-528(1981)

[3] F.O. Speck. Mixed Boundary Value Problems Of the Type of Sommerfeld's Half- Plane problem. Proc of the Royal Society of Edinburg, 104., p.p. 261-277(1986)

[4] V.G. Daniel . On the Solutionof two Coupled Winer-Hopf Equations. Siam J. Appl. Math., 44., p.p. 667-680(1984

[5] S. A. Hosseini Matikolai. Solvability of the Boundary Value Problem Coordinated with the Anisotropic Helmholtz-Shrodinger Equation. Word Applied science Journal, 11.(11.), 13481352(2010)

[6] S. A. Hosseini Matikolai. Solvability of the Boundary Value Problem Coordinated with the Anisotropic Helmholtz-Shrodinger Equation in case of $k_{+}=k_{-}$. World Alpplied sciences Journal, [Accepted] 11.(11), (2011).

[7] S. A. Hooseini Matikoni On the Structure of Wiener Operation Corresponting to the Anisotropic Boundary Value Connected with Helmhoitz Shrodinger equation with boundaru conditions of the first and second type. Proc. Of the Yerevan State University, No2., pp 22_26 (2011)

[8] G.i. Eskin Boundary Value Problems for Ellipit Pseudodifferntinl Equntican Mathematical Society, Providence, RI, P,p 283-300(1981) (in Russino1973)

[9 ] F.O. Speck Sommerfel Diffraction Problems with First and Second kind Boundary Condition Society for industrial and Applied Mathematics, 20 (2.), p.p. 396-407 (1989)

[10] A.E. heins. The Somerfeld Half- Plane Problem Revisted. The Factoring of a Matrix of Analytics Functions. Mah. Methods Appl. Sci., 5, p.p.14-24 (1983)

[11] N.I. maskhelishvili Singular integral Equations, Nauka, Moscow, p.p.126-140 (1968)

[12] F.D. Gakhov. Boundary Problems, Fizmation, Moscow, 221-243 (1963) 
S.A. Hosseini Matikolai, H. Jafari, Roshanak Lotfikar/ TJMCSVol. 4 №. 3 (2012) 301 - 309

[13] G.S. Litvinchnk, I.M.Spitkovskii. Factorizon of Measurable Matrix Functions, Akademie Verlag Berlin, 182-196 (1987)

[14] K. Glancey and I. Gohberg. Factorizaton of Matrix Functions and Sigular integral Operators, Advanced and Appl, Birkhauser, Basel, 211-230(1981) 